\title{
R-related quantities in QCD and the Adler D-function
}

\author{
Olga Solovtsova* \\ Gomel State Technical University, 246746, Gomel, Belarus \\ Bogoliubov Laboratory of Theoretical Physics, JINR, 141980, Dubna, Russia \\ E-mail: olsolatheor.jinr.ru
}

\begin{abstract}
From the point of view of the quark-hadron duality, we consider a description of physical quantities and functions that are defined through the function $R(s)$, the normalized hadronic crosssection, and connected with the Adler $D$-function. This function defined in the spacelike region is a smooth function without traces of the resonance structure and one can expect that reflects more precisely the quark-hadron duality and will be a convenient object for comparing theoretical predictions with experimental data. Based on the $D$-function in the theoretical description, we find good agreement between our results and the corresponding experimental data down to the lowest energy scale that is not calculable within the standard perturbative QCD. We investigate the reason of such good agreement and, as a result, we formulate a criterion which we call the $R$ $-D$ self-duality.
\end{abstract}

XXI International Baldin Seminar on High Energy Physics Problems, September 10-15, 2012

JINR, Dubna, Russia

${ }^{*}$ Speaker. 


\section{Introduction}

To compare theoretical results and experimental data one often uses the concept of quark-hadron duality which establishes the bridge between quarks and gluons, a language of theoreticians, and real measurements with hadrons performed by experimentalists.

The idea of quark-hadron duality was formulated by E. Poggio, H. Quinn, and S. Weinberg [1] as follows: Inclusive hadronic cross sections, once they are appropriately averaged over an energy interval, must approximately coincide with the corresponding quantities derived from the quarkgluon picture. There are various areas of hadronic physics dealing with different manifestations of quark-hadron duality (see, e.g., Refs. [2, 3]).

In this work, we concentrate on physical quantities and functions that are defined through the function $R(s)$, the normalized cross-section for the process $e^{+} e^{-}$annihilation into hadrons, integrated with some other functions. By definition, all these quantities and functions include an infrared region as part of the interval of integration and, therefore, they cannot be directly calculated within perturbative quantum chromodynamics.

Clearly, it will be fruitful to connect measured quantities with the "simplest" theoretical objects. Some single-argument functions which are directly connected with experimentally measured quantities can play the role of these objects. The cross-section for the process of $e^{+} e^{-}$annihilation into hadrons, or the function $R(s)$, is a physically measured quantity defined for timelike momentum transfer, but at the low energy scale has a resonance structure which is difficult to describe without model considerations. At the same time, the Adler $D$-function connected with $R(s)$ and defined in the Euclidean region for $Q^{2}=-q^{2}>0$ is a smooth function without traces of the resonance structure. Therefore, one can expect that the Adler function will be useful and it will reflect more precisely the quark-hadron duality as compared, for example, with the smeared quantity $R_{\Delta}\left(q^{2}\right)$ [1] defined in the timelike region.

Let us remind that in $1974 \mathrm{~S}$. Adler [4] observed that through $R(s)$ is measured in the timelike region, the natural place to compare experiment with scaling predictions of various theories in QCD is the spacelike region and, consequently, he suggested to use the function $T(-s)$ which is the first derivative of the function $R(s)$. Two years later A. De Rújula and H. Georgi [5] used a modified version of this idea defining $D(s)=-s T(-s),-s=Q^{2}>0$ (the spacelike region). This function $D$ has been revived under the name of the "Adler function". The $D$-function was studied in a number of papers $[6,7,8,9,10,11,12,13,14,15,16,17,18]$ (see Ref. [19] for more details). We investigate the reason of such good agreement and as a result we formulate a criterion which we call as the $R-D$ self-duality.

In this report, we concentrate on the $D$-function, the hadronic contribution to the anomalous magnetic moment of the leptons, and the hadronic contribution to the electromagnetic coupling. The approach that we consider here to describe above quantities is based on the variational perturbation theory (VPT) [20, 21]. The structure of VPT series is different from the structure of conventional PT-expansion. VPT, in the case of QCD, leads to a new small $a$-expansion parameter [20]. This parameter obeys a special equation whose solutions are always smaller than unity for any value of the original coupling constant, that allows us to go beyond the weak-coupling regime and deal with considerably lower energy scales than in the case of PT. As has been demonstrated in Ref. [9], the "experimental" $D$-functions corresponding to the $e^{+} e^{-}$annihilation into hadrons and 
the inclusive hadronic $\tau$-decays in the vector channel both are in good agreement with behavior obtained in the framework of the nonperturbative $a$-expansion method. Note that any finite order of the operator product expansion (OPE) fails to describe the infrared tail of the $D$-function. The model which is based on the VPT and involves into consideration a summation of threshold singularities [22] was reported at the Baldin seminar in 2006 by I. Solovtsov [23]. It was demonstrated that the proposed method allows to describe these quantities well. Note the method based on the analytic perturbation theory [24] leads to very close results [25]. Continuing these researches we concentrate on the reason of such good agreement.

\section{Some numerical results}

The hadronic contribution to the lepton anomalous magnetic moment in the leading order in the electromagnetic coupling constant is defined by

$$
a_{l}^{\mathrm{had}}=\frac{1}{3}\left(\frac{\alpha}{\pi}\right)^{2} \int_{0}^{\infty} \frac{d s}{s} K_{l}(s) R(s),
$$

where $\alpha^{-1}=\alpha(0)^{-1}=137.03599911(46), \quad l=\mu, \tau, e$, and $K_{l}(s)$ is a known the vacuum polarization factor. As a result of calculations we obtain values:

$$
a_{\mu}^{\mathrm{had}}=(702 \pm 16) \times 10^{-10}, a_{e}^{\mathrm{had}}=1.65 \times 10^{-12}, a_{\tau}^{\mathrm{had}}=3.30 \times 10^{-6} .
$$

The hadronic correction to the electromagnetic fine structure constant at the $Z$-boson scale, $\Delta \alpha_{\mathrm{had}}^{(5)}(s)$, can be represented in the form of the dispersion integral

$$
\Delta \alpha_{\mathrm{had}}^{(5)}\left(M_{Z}^{2}\right)=-\frac{\alpha(0)}{3 \pi} M_{Z}^{2} \mathscr{P} \int_{0}^{\infty} \frac{d s}{s} \frac{R(s)}{s-M_{Z}^{2}} .
$$

Our calculation gives the result: $\Delta \alpha_{\text {had }}^{(5)}\left(M_{Z}^{2}\right)=(279.9 \pm 4.0) \times 10^{-4}$ which close to value extracted from a wide range of data describing $e^{+} e^{-} \rightarrow$ hadrons: $\Delta \alpha_{\mathrm{had}}^{(5)}\left(M_{Z}^{2}\right)=\left(275.5 \pm 1.9_{\text {expt }} \pm 1.3_{\text {rad }}\right) \times$ $10^{-4}[26]$.

Let us turn to the hadronic correction to the muon. The expression (2.1) can be rewritten in terms of the $D$-function as follows [27]

$$
a_{\mu}^{\mathrm{had}}=\frac{1}{6}\left(\frac{\alpha}{\pi}\right)^{2} \int_{0}^{1} \frac{d x}{x}(1-x)(2-x) D\left(\frac{x^{2}}{1-x} m_{\mu}^{2}\right) .
$$

It is should be emphasized that the expressions (2.1) and (2.4) are equivalent due to the analytic properties of the correlator $\Pi\left(q^{2}\right)$. If one uses a method that does not maintain the required properties, expressions (2.1) and (2.4) will no longer be equivalent and will imply different results. This situation is similar to that which occurs in the analysis of inclusive $\tau$-decay [28]. Within VPT this transformation is justified [29], and one can use equally well either the expression (2.1) or the expression (2.4). 


\section{3. $R-D$ self-duality}

Now, we investigate the question: What we can say about connection between kernels in (2.1) and (2.4) when these expressions are equivalent? Let us rewrite these expressions in a more general form:

$$
Q_{M}=\int_{0}^{\infty} \frac{d s}{s} M(s) R(s),
$$

for the Minkowskian (timelike) region and

$$
Q_{E}=\int_{0}^{\infty} \frac{d t}{t} E(t) D(t),
$$

for the Euclidean (spacelike) region. The $R-D$ self-duality means

$$
Q_{M}=Q_{E}
$$

The answer to the question is connection between the kernels

$$
M(s)=s \int_{0}^{\infty} d t \frac{E(t)}{(s+t)^{2}} .
$$

According to this expression $M(s)$ is an analytic function in the complex plane with a cut along the negative real axis. The inverse relation reads

$$
E(t)=-\frac{1}{2 \pi \mathrm{i}} \int_{t-\mathrm{i} \varepsilon}^{t+\mathrm{i} \varepsilon} \frac{d z}{z} M(-z) .
$$

Applying this result to the hadronic correction to the muon, we get $R-D$ self-duality expressions:

$$
\begin{aligned}
& a_{\mu}^{\mathrm{had}}=\frac{1}{3}\left(\frac{\alpha}{\pi}\right)^{2} \int_{0}^{\infty} \frac{d s}{s} M(s) R(s), \\
& a_{\mu}^{\mathrm{had}}=\frac{1}{3}\left(\frac{\alpha}{\pi}\right)^{2} \int_{0}^{\infty} \frac{d t}{t} E(t) D(t),
\end{aligned}
$$

where

$$
\begin{gathered}
M(s)=\int_{0}^{1} d x \frac{x^{2}(1-x)}{x^{2}+(1-x) s / m_{\mu}^{2}}= \\
\eta^{2}\left(1-\frac{\eta^{2}}{2}\right)+(1+\eta)^{2}\left(1+\frac{1}{\eta^{2}}\right)\left[\ln (1+\eta)-\eta+\frac{\eta^{2}}{2}\right]+\frac{1+\eta}{1-\eta} \eta^{2} \ln \eta \\
E(t)=\frac{1}{2}\left[\frac{\sqrt{1+4 m_{\mu}^{2} / t-1}}{\sqrt{1+4 m_{\mu}^{2} / t+1}}\right]^{2}, \\
\eta=\frac{1-v}{1+v}, \quad v=\sqrt{1-\frac{4 m_{\mu}^{2}}{s}} .
\end{gathered}
$$




\section{Summary}

A method of performing QCD calculations in the nonperturbative domain was used. This method is based on the variational perturbation theory in $\mathrm{QCD}$, takes into account the summation of threshold singularities and the involvement of nonperturbative light quark masses. We demonstrated that this method allows us to describe $R$-related quantities rather well. We investigated the reason of such good agreement and, as a result, we formulated a criterion which we called the $R-$ $D$ self-duality.

It is a pleasure to thank A.L. Kataev and O.V. Teryaev for interest in the work and useful discussions. This work was supported in part by the BelRFBR-JINR grant No. F12D-002 and the RFBR grant No. 11-01-00182.

\section{References}

[1] E.C. Poggio, H.R. Quinn, and S. Weinberg, Phys. Rev. D 13 (1976) 1958.

[2] W. Melnitchouk, R. Ent, and C. Keppel, Phys. Rept. 406 (2005) 127.

[3] W. Lucha and D. Melikhov, Phys. Rev. D 73 (2006) 054009.

[4] S.L. Adler, Phys. Rev. D 10 (1974) 3714.

[5] A. De Rújula, H. Georgi, Phys. Rev. D 13 (1976) 1296.

[6] S. Peris, M. Perrottet, and E. de Rafael, JHEP 05 (1998) 011.

[7] S. Eidelman, F. Jegerlehner, A.L. Kataev, and O. Veretin, Phys. Lett. B 454 (1999) 369.

[8] K.A. Milton, I.L. Solovtsov, and O.P. Solovtsova, Phys. Rev. D 64 (2001) 016005.

[9] A.N. Sisakian, I.L. Solovtsov, and O.P. Solovtsova, JETP Lett. 73 (2001) 166.

[10] A.E. Dorokhov, Phys. Rev. D 70 (2004) 094011.

[11] A.L. Kataev, Phys. Lett. B 668 (2008) 350.

[12] F. Jegerlehner, Nucl. Phys. Proc. Suppl. 181-182 (2008) 135.

[13] P.A. Baikov, K.G. Chetyrkin, and J.H. Kühn, Phys. Rev. Lett.101 (2008) 012002.

[14] A.V. Nesterenko, Nucl. Phys. Proc. Suppl. 186 (2009) 207.

[15] B.A. Magradze, Few Body Syst. 48 (2010) 143 [Erratum-ibid. 53 (2012) 365.

[16] A.L. Kataev and S.V. Mikhailov, PoS QFTHEP2010 (2010) 014.

[17] T. Goecke, C.S. Fischer, and R. Williams, Phys. Lett. B 704 (2011) 211.

[18] P.A. Baikov, K.G. Chetyrkin, J.H. Kühn, and J. Rittinger, Phys. Lett. B 714 (2012) 62.

[19] S.L. Adler, "Adventures in theoretical physics: Selected papers of Stephen L. Adler: Drafts of commentaries", hep-ph/0505177, $117 \mathrm{pp}$.

[20] I.L. Solovtsov, Phys. Lett. B 327 (1994) 335; 340 (1994) 245.

[21] A.N. Sissakian and I.L. Solovtsov, Phys. Part. Nucl. 30 (1999) 461.

[22] K.A. Milton and I.L. Solovtsov, Mod. Phys. Lett. A 16 (2001) 2213.

[23] I.L. Solovtsov, Proceedings of the XVIII International Baldin Seminar on High Energy Physics Problems, Eds. A.N. Sissakian, V.V. Burov, A.I. Malakhov, JINR E1,2-2008-113, Vol. I, p. 28.

[24] D.V. Shirkov and I.L. Solovtsov, Phys. Rev. Lett. 79 (1997) 1209.

[25] K.A. Milton, I.L. Solovtsov, and O.P. Solovtsova, Mod. Phys. Lett. A 21 (2006) 1355. 
[26] K. Hagiwara, et al., Phys. Rev. D 69 (2004) 093003.

[27] E. de Rafael, Phys. Lett. B 322 (1994) 239.

[28] K.A. Milton, I.L. Solovtsov and O.P. Solovtsova, Phys. Lett. B 415 (1997) 104.

[29] H.F. Jones and I.S. Solovtsov, Phys. Lett. B 349 (1995) 519. 